\title{
Il verbo deficitare: un deficit dei vocabolari dell'italiano?
}

\author{
Raffaella Setti
}

PUBBLICATO: 28 SETTEMBRE 2021

\section{Quesito:}

Alcuni lettori ci chiedono se "esista" in italiano il verbo deficitare; alcuni si domandano se si possa al suo posto usare il latinismo deficere. Un lettore domanda se deficitario e deficiente siano sinonimi, mentre altri chiedono il perché della presenza della $i$ in deficiente e deficienza.

\section{Il verbo deficitare: un deficit dei vocabolari dellitaliano?}

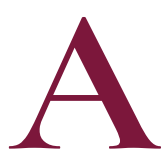

1la domanda espressa nel titolo si può rispondere che è proprio così per il momento i principali dizionari dell'uso dell'italiano (Vocabolario Treccani, GRADIT, Devoto-Oli, Zingarelli 2020) non registrano la forma deficitare di cui, in realtà, iniziano a esserci numerose attestazioni nella lingua corrente con significato di 'mancare, essere carente'. Impostando una ricerca su Google (pagine in italiano, 2I/I/2O2I) si rileva questa situazione: per l'infinito deficitare 2.7IO occorrenze (alcuni esempi: "i beni primari di cui un Paese non dovrebbe mai deficitare"; "le soluzioni continuano a deficitare"; "potrebbero deficitare del giusto apporto di questa vitamina"); per le forme flesse abbiamo verificato: deficitano 5.510 ("deficitano di polso"; "I tutorial deficitano un po' in agosto"; "tanti smartphone deficitano sul livello sonoro"; "i campioni normalmente deficitano di ferro"); deficita I3.200 ("il clacson deficita un po"; "lei deficita di logica o ha bevuto"; "deficita un po' di umorismo"; "se l'atto deficita di questi elementi"; "deficita di materiale scolastico e di un'infermeria"); deficitiamo 688 ("noi italiani deficitiamo in capacità di narrare"; "a criticare son tutti bravi, a proporre deficitiamo tutti (scusate il deficitiamo, ma mi viene cosi)"; "condizione fisica di cui deficitiamo per motivi anagrafici"). Una situazione dunque che, nel suo insieme, attesta non solo una certa diffusione del verbo, ma l'avvenuta integrazione nella morfosintassi con la presenza delle diverse forme con prevalenza, com'è naturale, delle terze persone.

Molto più contenute le presenze sui giornali: nell'archivio della "Repubblica" si rintracciano solo tre occorrenze di deficitano di cui la prima del 24/8/2006 ("tra gli spettacoli deficitano i grossi nomi del panorama musicale"); soltanto una di deficita in un articolo sportivo in cui viene notato, con un uso assoluto del verbo che ne sottolinea anche il tono ironico, che "Milinkovic deficita" (II/Ir/2Org). Nell'archivio del "Corriere" deficitare ricorre una sola volta in un articolo del I7/5/2018 ("oltre a deficitare di una rivoluzione francese [...] l'universo mondo musulmano pendola fra più interpretazioni di quelle scritture") mentre le altre forme coniugate non compaiono mai.

Giustamente alcuni dei nostri interlocutori si e ci domandano come mai in italiano sia entrata solo la forma latina deficit e non il verbo deficere da cui deriva. In effetti, il verbo latino, di cui deficit è la terza persona singolare del presente indicativo ('manca'), passa in italiano soltanto come voce dotta con impieghi rari ed esclusivamente letterari; non si radica nella lingua comune in cui lo stesso significato è veicolato da numerosi sinonimi (mancare, difettare, scarseggiare, non bastare, essere carente) e lo troviamo ancora circolante nel linguaggio corrente ormai quasi soltanto grazie alla famosa sentenza latina melius abundare quam deficere, peraltro spesso citata solo nella prima parte melius abundare..., con sfumature di significato variabili a seconda dei cotesti in cui la si pronuncia. 
La parola deficit, base del verbo deficitare, si è però diffusa come sostantivo maschile (il deficit), ed è entrata in italiano attraverso il francese, dove figurava almeno dal 1798 all'interno di inventari per indicare articoli mancanti (come segnalato nel Trésor de la langue française informatisé: "Il y a plusieurs déficit dans cet inventaire",Ac. I798-г878); con la stessa funzione, nella forma abbreviata def. (deficit), la ritroviamo come formula negli apparati critici (tradizionalmente scritti in latino) dei testi manoscritti per segnalare parti mancanti di testo, con il valore letterale di 'è privo, manca (di una sezione di testo)'.

La prima diffusione del termine in Italia è probabilmente avvenuta nel periodo immediatamente precedente alla Rivoluzione, in cui circolavano frequenti e insistenti notizie sulla situazione critica delle finanze francesi, afflitte appunto da un grave deficit. Lo Zingarelli data infatti la parola al I783, mentre il primo esempio riportato nel GDLI, col significato etimologico di 'carenza, scarsità', è tratto dalle Novelle di Domenico Luigi Batacchi, r79r: "E ognun pensa fra sé: Quand'ella torna / il Re non avrà deficit di corna!"); ritroviamo poi la parola, nell'accezione economica, ripetuta più volte in uno scritto di Giuseppe Mazzini del I 842 dedicato alla cerimonia per l'Anniversario della scuola italiana gratuita in Londra in cui a più riprese si insiste sui problemi di bilancio dell'istituto (si cita dalle Opere, ed. a cura di Luigi Salvatorelli, II, Scritti, Milano, Rizzoli, I956):

Dichiarò il deficit con volto d'uomo che confida nell'aiuto dei buoni e depositando lo Stato Generale dell'Amministrazione nelle mani de' sottoscrittori, offrì a qualunque volesse sincerarsi dell'esattezza, comunicazione dei documenti (p. 332);

Esiste un deficit di lire 50 [...] come risulta dallo Stato Generale dell'Amministrazione della Scuola (p. 346);

Ah! se noi non credessimo che nell'altro mese potremo annunziare cancellato quel deficit ai nostri lettori, dispereremmo, non dell'Italia [...] ma dell'attuale generazione Italiana (p. 348).

La parola, tuttavia, non deve aver avuto immediata e favorevole accoglienza, visto che il suo primo ingresso in un vocabolario non solo è avvenuto dopo quasi un secolo (nel I860 nel Vocabolario di parole e modi errati di Filippo Ugolini), ma è stato accompagnato da un commento poco lusinghiero:

parlandosi di conti e di amministrazioni, pare che alcuni non possano fare a meno di non usare di questo latinismo, a cui però si può ben supplire con le parole 'mancanza, manco, scemamento'.

Non si può tuttavia escludere che il termine circolasse, sicuramente in latino, già alla fine del Cinquecento, seppure come verbo, nel significato generico di 'mancare': in questo senso ci indirizzano le Eleganze insieme con la copia della lingua toscana, e latina scelte e compilate da Aldo Manuzio (Roma, I589) che, alla voce Diminuire, ci offrono una preziosa testimonianza del traducente consigliato per la forma latina deficit, resa appunto con i verbi menovare e mancare:

La tua facoltà si va di dì in dì diminuendo, si và menovando, và scemandosi, calando, mancando, scapitando, deteriorando, consumandosi, minor diventando, và di mal'in peggio, non solo non riceve accrescimento, ma più tosto và perdendo del capitale

periodo seguito dalla versione latina:

Res tua in dies deterior fit, imminuitur, deficit, consumitur [...] corroditur, deficit, dilapidatur: fortune tuae in dies aliquot detrimenti capiunt [...].

Da notare anche che già qui il contesto è riconducibile all'ambito dell'economia, alla "perdita di capitale", proprio quello in cui il termine si affermerà nel suo significato tecnico di 'ammanco di 
bilancio' e dove tuttora resta purtroppo molto frequente a indicare il disavanzo del bilancio dello Stato.

Più tarda, ai primi del Novecento, l'estensione del termine al linguaggio della medicina, altro settore in cui si è specializzato e radicato: in ambito medico deficit indica una 'diminuzione dell'attività funzionale dell'organismo o di determinati organi' (Vocabolario Treccani) e si parla quindi di deficit visivo, cardiaco, respiratorio, e in psicopatologia si definisce il deficit intellettivo l'indebolimento transitorio o permanente delle facoltà intellettive'.

Il verbo deficitare si è formato recentemente secondo una modalità molto produttiva e ricorrente nell'italiano anche contemporaneo: la conversione, per cui si può passare da un verbo a un nome (del tipo cambiare > cambio; disegnare > disegno; saltare > salto) o, viceversa, da un nome a un verbo (del tipo maschera $>$ mascherare; sale $>$ salare; trucco $>$ truccare). In questo caso, come per molti altri nuovi verbi, deficitare è il risultato di una conversione da un nome a un verbo per cui, sulla base nominale latina deficit, si è aggiunta la desinenza -are della prima coniugazione. Per questo genere di formazioni talvolta è difficile stabilire la direzione del processo (dal verbo o dal nome?), ma per deficitare troviamo conferma nei dizionari che, come abbiamo visto, registrano almeno dalla seconda metà dell'Ottocento il latinismo deficit, ma ancora oggi non riportano il verbo. Questo ci rivela che la conversione si è svolta a partire dal nome a cui è stata applicata la desinenza -are della prima coniugazione verbale dell'italiano.

Si tratta dello stesso meccanismo con cui, a partire da basi nominali straniere, si sono formati negli ultimi anni, ad esempio, i verbi chattare, twittare, whatsappare, crashare (sulla produttività di tale processo si veda anche questa scheda), e molti altri ormai del tutto acclimatati nella lingua d'uso. Si ottengono cosi verbi adattati alla morfologia dell'italiano e quindi coniugabili come qualsiasi altro verbo della prima coniugazione. Tale modalità di formazione è senz'altro stata favorita dall'esigenza di simmetria tra sostantivo e verbo corrispondente: così come per mancanza c'è mancare, per scarsitá scarseggiare, per perdita perdere, anche per deficit si è ricostruita una forma verbale coniugabile, visto che ormai era andato perduto il legame con verbo latino originario deficere. Come notato in apertura, le occorrenze del "nuovo" verbo non mancano, soprattutto in rete, e, visto il radicamento del sostantivo deficit in italiano, tra l'altro in settori come quelli dell'economia e della medicina che tendono a far filtrare nella lingua comune i loro termini, e la decisa tendenza verso formazioni verbali analoghe, possiamo ragionevolmente prevedere che anche deficitare entrerà nei futuri vocabolari. Quanto futuri è difficile dirlo.

Il verbo deficere non è sopravvissuto in italiano soltanto nella forma deficit, ma le sue tracce restano anche indeficiente edeficienza, voci dotte derivate rispettivamente dal participio presente latino deficiente $(m)$ 'carente, mancante' e dal sostantivo del latino tardo deficientia $(m)$ 'carenza, mancanza' (a sua volta formato su deficientem). In merito a queste forme, molte domande riguardano la grafia e la possibilità di eliminare la $i$ etimologica che, dal latino (in cui era presente), è arrivata fino a noi, e di scrivere quindi, cosi come si pronunciano, deficente e deficenza. Lo strumento di riferimento più autorevole e affidabile per questo genere di problemi è il DOP (Dizionario di Ortografia e Pronunzia, consultabile anche in rete), che alla voce deficiente indica come più corretta la grafia con $i$ (quindi deficiente), anche se registra, con il commento "meno bene", la forma deficente. Quindi non si tratta certo di un "grosso errore" e l'alternanza delle due grafie è giustificata dal fatto che la $i$ non ha nessuna motivazione fonetica, ma è un relitto della grafia latina, tipico delle parole di tradizione colta. Nel corso della storia della lingua italiana sono stati molti i tentativi di far prevalere criteri fonetici, quindi massima corrispondenza possibile tra grafia e pronuncia, rispetto alla permanenza di grafie etimologiche non più rispondenti all'effettiva pronuncia. E ormai non sono moltissimi in italiano i 
casi simili al nostro: possiamo citare almeno sufficiente, cielo e scienza. Se devo esprimere il mio parere, ammetto che mi trovo sostanzialmente in linea con le indicazioni del DOP, perché ritengo che vada garantita la trasmissione del lessico colto con le tracce storiche che ancora rivela, resistendo alla tentazione di appiattire tutto sulla dimensione della contemporaneità. Forse proprio quella $i$ di deficiente/deficienza ha incuriosito i nostri interlocutori e offerto l'occasione di questo approfondimento che ci auguriamo aiuti anche le nuove generazioni a comprendere la motivazione di questa grafia, che cosi, forse, diventerà più facile da ricordare.

Se la grafia di deficiente è rimasta sostanzialmente invariata nel tempo, non si può dire la stessa cosa per quel che riguarda il significato. Attestato in italiano fin dal Trecento nel significato originario di 'mancante, insufficiente', con diverse sfumature fino a 'difettoso, imperfetto', attraverso la mediazione dell'inglese, nel XIX secolo ha assunto l'accezione medica di 'carente nelle funzioni fisiche o mentali', come eufemismo di frenastenico quando riferito ai fanciulli. Per l'italiano, una delle prime attestazioni che sono riuscita a rintracciare (con l'aiuto del mio dottorando Stefano Miani, che ringrazio) è del I899 ed è contenuta nell'intitolazione della Lega Nazionale per la protezione dei fanciulli deficienti fondata a Roma dallo psichiatra Clodomiro Bonfigli; il testo retrodata di qualche anno la lessicografia che registra il termine a partire dal Dizionario moderno delle parole che non si trovano nei dizionari comuni di Alfredo Panzini (1905), dove si legge: "eufemismo che talora, specie parlando di fanciulli equivale a 'frenastenico". A distanza di un ventennio il termine doveva però essere già diffuso nella lingua comune come offesa, se nei Neologismi buoni e cattivi (di G. Rigutini-G. Cappuccini del I926) si notava: "Deficiente, passando dal linguaggio della medicina in cui vale 'scarso, privo' (di mente, d'alcune facoltà mentali) a quello dell'improperi: Va' là che sei un povero deficiente".

È probabile che proprio questo slittamento semantico di deficiente, dal lessico tecnico medico a insulto nella lingua comune, abbia favorito l'affermazione di un altro tecnicismo che, sempre dalla stessa base latina deficit, assumesse il valore univoco che deficiente stava perdendo. Arriviamo cosi a deficitario, calco del francese déficitaire nel significato finanziario di 'in perdita', attestato in italiano dal I927 (cfr. l'Etimologico), mentre sembrerebbe possibile retrodatare almeno al I9I3 l'accezione psichiatrica di 'carente di facoltà mentali', grazie a Google libri che restituisce unoccorrenza in "Psiche" Rivista di Studi Psicologici (vol. II, p. I7I): «stato "deficitario" puro dai sintomi accidentali, come allucinazioni, agitazione, confusione, ecc.». Nella lingua attuale deficitario mantiene questi due ambiti d'uso prevalenti, l'economia (con sconfinamenti nella politica, governo deficitario, azione politica deficitaria, ecc.) e la medicina, in cui corrisponde a 'insufficiente, carente' (es. alimentazione deficitaria), anche se si nota una tendenza a un'estensione nell'uso dell'aggettivo (soprattutto in espressioni generiche come "situazione deficitaria", "stato deficitario", "rapporto deficitario"), forse perché sentito come più tecnico rispetto ai suoi numerosissimi sinonimi.

A tale proposito, un'ultima osservazione rispetto alla possibilità di scambio tra deficitario e deficiente: dal punto di vista etimologico i due aggettivi sono sostanzialmente sinonimi, ma nella selezione dell'uno o dell'altro dobbiamo tenere ben presente anche il valore che ciascuno di essi ha assunto nell'uso e nella comunicazione. Dato che deficiente rientra ormai anche nel repertorio degli insulti è opportuno fare molta attenzione al contesto in cui lo si inserisce, evitando quindi qualsiasi riferimento a persone e precisando il tipo di deficienza rilevata. Dove questo non sia possibile o resti un margine di ambiguità, meglio ricorrere a deficitario o a uno dei molti sinonimi a disposizione come carente, insufficiente, mancante, scarso. 


\section{Cita come:}

Raffaella Setti, // verbo deficitare: un deficit dei vocabolari dell'italiano?, "Italiano digitale", XVIII, 2021/3 (luglio-settembre)

DOI: 10.35948/2532-9006/2021.11607

Copyright 2021 Accademia della Crusca

Pubblicato con licenza creative commons CC BY-NC-ND 